\title{
ACF7 Is a Hair-Bundle Antecedent, Positioned to Integrate Cuticular Plate Actin and Somatic Tubulin
}

\author{
Patrick J. Antonellis, ${ }^{1,2 *}$ Lana M. Pollock, ${ }^{1,3 *}$ Shih-Wei Chou, ${ }^{1,2 *}$ Ahmed Hassan, ${ }^{5}$ Ruishuang Geng, ${ }^{1}$ Xi Chen,,${ }^{1,2}$ \\ Elaine Fuchs, ${ }^{6}$ Kumar N. Alagramam, ${ }^{1,3,4}$ Manfred Auer, ${ }^{5}$ and Brian M. McDermott Jr ${ }^{1,2,3,4}$ \\ Departments of ${ }^{1}$ Otolaryngology-Head and Neck Surgery, ${ }^{2}$ Biology, ${ }^{3}$ Genetics and Genome Sciences, and ${ }^{4}$ Neurosciences, Case Western Reserve University, \\ Cleveland, Ohio 44016, ${ }^{5}$ Life Sciences Division, Lawrence Berkeley National Laboratory, Berkeley, California 94720, and ${ }^{6}$ Howard Hughes Medical Institute, \\ The Rockefeller University, New York, New York 10065
}

The precise morphology of the mechanosensitive hair bundle requires seamless integration of actin and microtubule networks. Here, we identify Acf7a (actin crosslinking family protein 7a) as a protein positioned to bridge these distinct cytoskeletal networks in hair cells. By imaging Acf7a-Citrine fusion protein in zebrafish and immunolabeling of vestibular and cochlear mouse hair cells, we show that Acf7a and ACF7 circumscribe, underlie, and are interwoven into the cuticular plate (CP), and they also encircle the basal body of the kinocilium. In cochlear hair cells, ACF7 localization is graded, with the highest concentration near each fonticulus - an area free of F-actin in the region of the $\mathrm{CP}$ that contains the basal body. During hair-cell development and regeneration, Acf7a precedes formation of the hair bundle and CP. Finally, electron tomography demonstrates that the ends of microtubules insert into the $\mathrm{CP}$ and are decorated with filamentous linkers connecting microtubules to the CP. These observations are consistent with ACF7 being a linker protein, which may shape the cytoskeleton of the hair cell early during hair-bundle genesis.

Key words: actin; hair cell; hearing; mechanotransduction; microtubule; zebrafish

\section{Introduction}

Mechanosensory hair cells of the inner ear are responsible for transduction of sound stimuli and head movements to enable the senses of hearing and balance, respectively. Protruding from the apical surface of the hair cell is the hair bundle, which consists of stereocilia of increasing height that hold extraordinarily sensitive mechanically gated channels (Hudspeth, 1982). Development of stereocilia is preceded by the formation of a microtubule-based kinocilium, which has the basal body at its root (Tilney et al., 1992).

The actin-based cuticular plate (CP) is thought to act as a substratum on which stereocilia pivot (DeRosier and Tilney,

Received May 3, 2013; revised Nov. 1, 2013; accepted Nov. 7, 2013.

Author contributions: P.J.A., L.M.P., S.-W.C., A.H., M.A., and B.M.M. designed research; P.J.A., L.M.P., S.-W.C., A.H., R.G., X.C., M.A., and B.M.M. performed research; E.F. contributed unpublished reagents/analytic tools; P.J.A., L.M.P., S.-W.C., A.H., R.G., X.C., E.F., K.N.A., M.A., and B.M.M. analyzed data; P.J.A., L.M.P., S.-W.C., A.H., R.G., E.F., K.N.A., M.A., and B.M.M. wrote the paper.

This research was supported by National Institutes of Health (NIH) Grants DC009437 (B.M.M.), DC010816 (K.N.A.), and GM051487 (M.A., A.H.), NIH Training Grant GM008056-28 (L.M.P.), and the Center for Clinical Research and Technology at University Hospitals (ase Medical Center (B.M.M.). E.F. is an Investigator of the Howard Hughes Medical Institute, and her ACF7 work is supported by NIH Grant R37 AR27883. The NIH Office of Research Infrastructure Program Award S10RR017980 supported research reported here. We are grateful to C. Fernando for zebrafish husbandry, X. Bai and N. Beckloff for assistance with producing and analyzing RNA-seq data, A. Althans for zebrafish phenotypic analyses, Z. Chen for assistance with neomycin treatment of larval zebrafish, J. Lee for preparation of samples, and the members of our laboratories for critically reviewing this manuscript.

*P.J.A., L.M.P., and S.-W.C. contributed equally to this work.

The authors declare no competing financial interests.

Correspondence should be addressed to Brian M. McDermott Jr, 11100 Euclid Avenue, Cleveland, $0 \mathrm{H} 44106$. E-mail:bmm30@case.edu.

DOI:10.1523/JNEUROSCI.1880-13.2014

Copyright $\odot 2014$ the authors $\quad 0270-6474 / 14 / 330305-08 \$ 15.00 / 0$
1989) and is linked in the soma by columns of microtubules oriented parallel to the longitudinal axis of the hair cell (Jaeger et al., 1994). Microtubules can also fan out from the basal body, which resides in the fonticulus - a region in the CP devoid of F-actin (Hawkins, 1976; Raphael et al., 1994; Raphael and Altschuler, 2003; Chacon-Heszele et al., 2012). The ends of the columnar microtubules are coated with a fuzzy cap of electrondense material and extend deep into the CP (Jaeger et al., 1994). Whether there are direct physical linkages that connect the CP to the microtubules remains unknown, as do the potential molecular linkers involved, should they exist.

Actin crosslinking family protein 7 (ACF7), encoded by the microtubule and actin crosslinking factor 1 (Macf1) gene, is an extremely large $(>500 \mathrm{kDa})$ cytoskeletal crosslinking protein that interacts with F-actin and microtubules to shape the morphology of cells (Leung et al., 1999; Suozzi et al., 2012). Microtubule and actin crosslinking factor 1 genes are known to be developmentally important because a null mutation in the mouse ortholog is embryonically lethal (Kodama et al., 2003; Chen et al., 2006), and, in the zebrafish, a mutation results in defects in animal-vegetal polarity of the oocyte and egg (Dosch et al., 2004; Gupta et al., 2010). ACF7 contains numerous protein domains to enable dynamic interaction with the cytoskeleton. Toward the $\mathrm{N}$ terminus, calponin homology 1 and 2 domains facilitate direct interaction with F-actin. Near the $\mathrm{C}$ terminus are the GAS2-related protein domain, which associates with microtubules and facilitates microtubule stabilization, the GSR (Gly-Ser-Arg)-repeat domain, which also interacts with microtubules, and the EF hand domains, which bind calcium (Wu et al., 2011; Suozzi et al., 2012). 

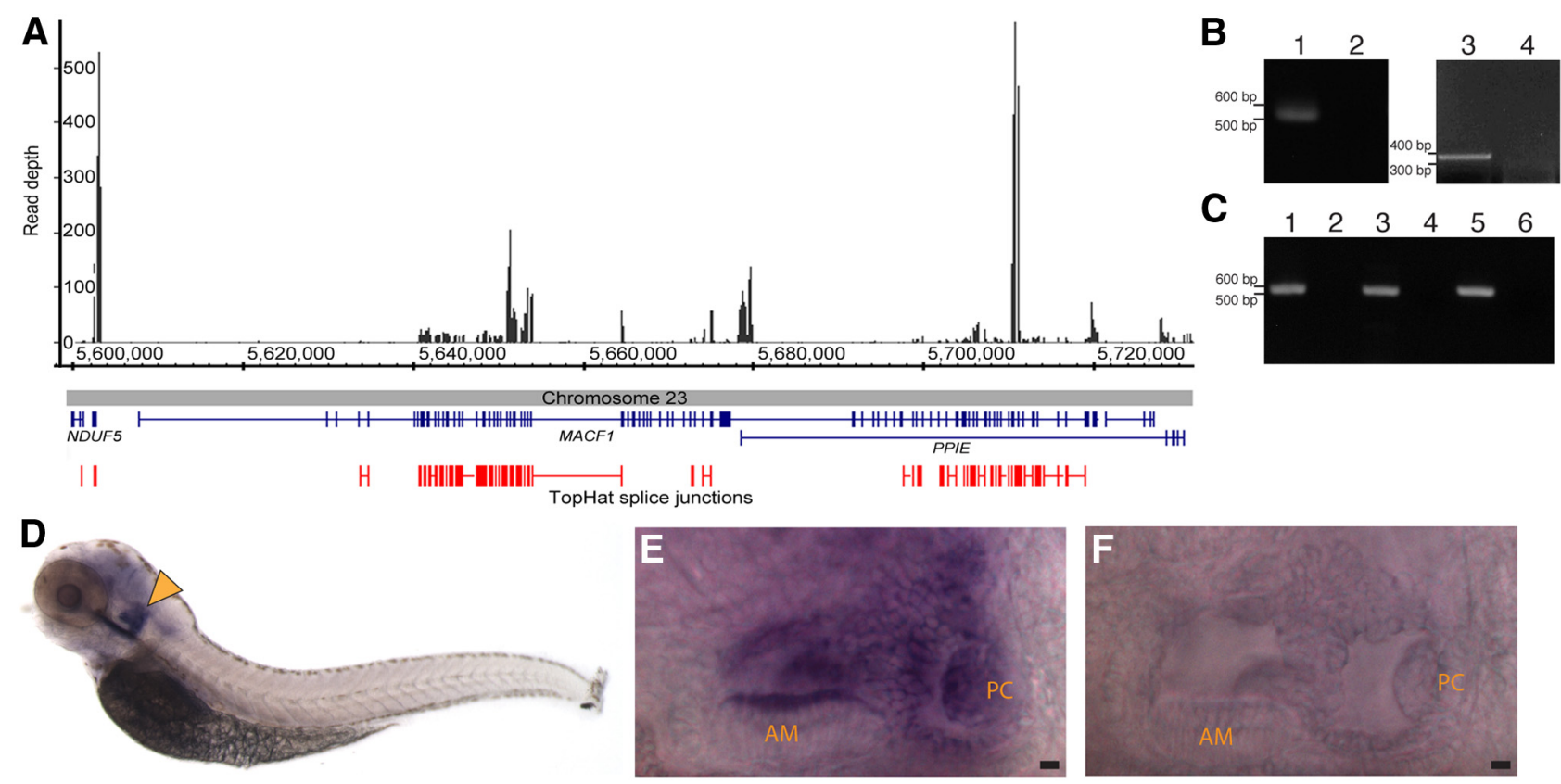

\section{G TTE SD |citrine CDNAISA TER}
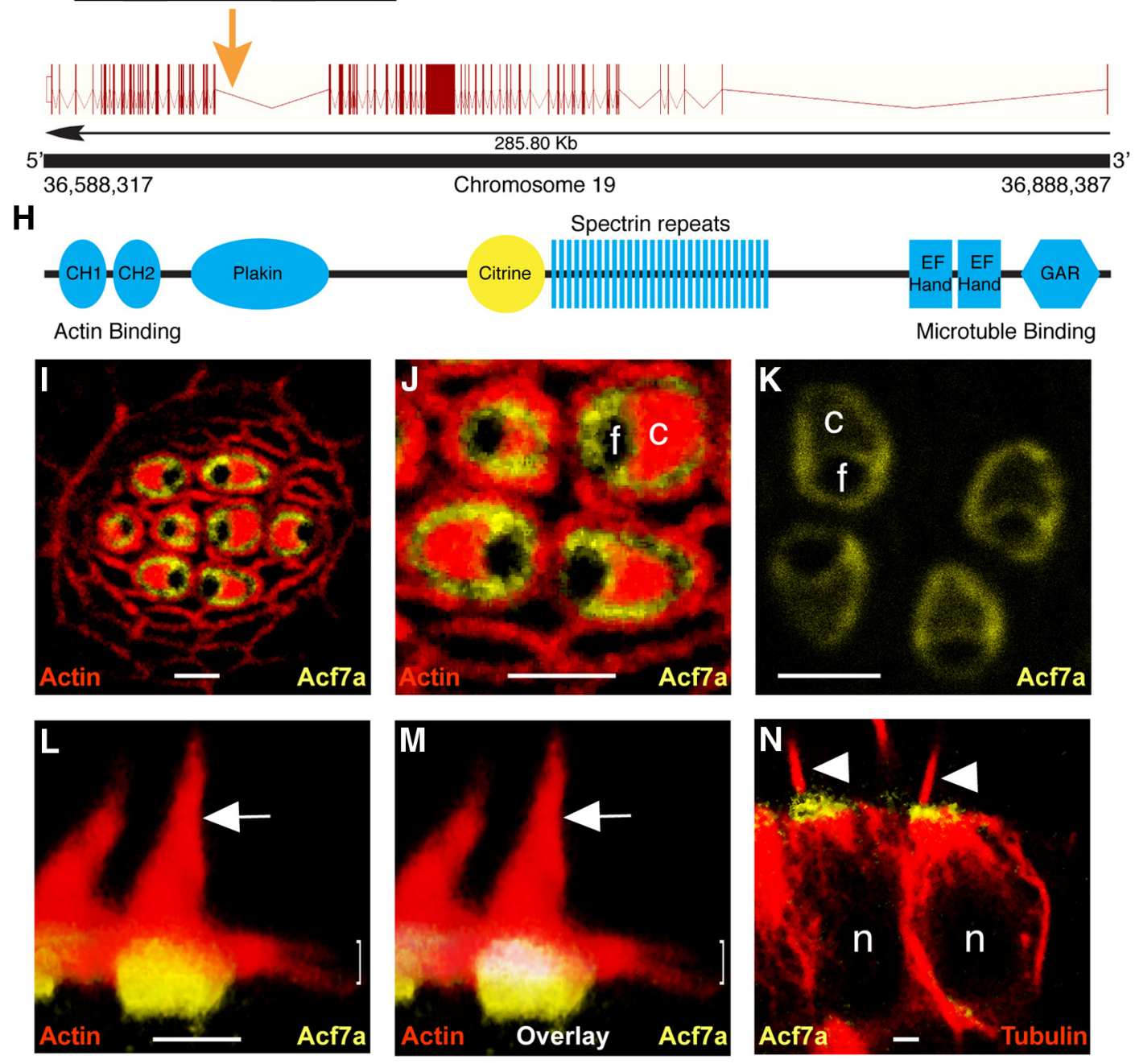

Figure 1. Expression of microtubule and actin crosslinking factor 1 genes in vertebrates and localization of Acf7a in zebrafish hair cells. A, Detection of MACF1 mRNA in chicken hair cells by RNA-seq. Depth of reads aligning to the chicken genome is displayed, with predicted splice junctions shown in red. Exons of the corresponding genes are displayed in blue. $\boldsymbol{B}$, RT-PCR demonstrates the presence of macfla mRNA [RT plus (lane 1) and RT minus control (lane 2)] and macf1b mRNA [RT plus (lane 3) and RT minus control (lane 4)] in zebrafish maculae. (Figure legend continues.) 
In this report, we identify ACF7 as a candidate molecular coupler, which is positioned to join $\mathrm{CP}$ actin and the somatic microtubule array of the hair cell.

\section{Materials and Methods}

Animals. Zebrafish strains Gt(macfla-citrine) $)^{c t 68 a /+}$ (Trinh le et al., 2011) and the wild-type strain Tübingen were used in these investigations. White Leghorn chickens and $F V B / N J$ mice were kept according to standard procedures. Animals of either sex were used.

Hair-cell isolation and RNA-seq. Chicken hair cells were isolated by picking with a glass micropipette (McDermott et al., 2007). RNA from 200 hair cells was isolated. Then, cDNA was synthesized, amplified (Ovation RNA-Seq System; NuGen), and sequenced using a HiSeq (Illumina) or a HiScan (Illumina).

The raw sequence data was assessed for quality using FASTQC software. Reads were aligned to the galGal3 reference genome with TopHat and provided to Cufflinks (Trapnell et al., 2010). Unannotated transcripts were annotated based on homologous sequences predicted using BLAST, with an E-value cutoff of $1 \times 10^{-5}$. The National Center for Biotechnology Information (NCBI) BLAST program uses $E$ values to indicate how strong an alignment is above alignment by random chance. Therefore, when we compared unannotated chicken RNA-seq transcript sequences using BLAST with the Swiss-Prot protein sequence database, we only considered strong alignments-those with $E$ values $<1 \times$ $10^{-5}$ - to reveal the potential chicken orthologs of a given gene. Expression values for each transcript were calculated as read counts. The alignment of reads was visualized using the Integrated Genome Browser through the Galaxy platform (Nicol et al., 2009).

$R T-P C R$. Macular tissue, from either adult mice or zebrafish, was isolated and used to generate cDNA (McDermott et al., 2007). The primer pairs used to amplify from mouse template cDNA were mus_macf1_1 forward (5' GAAGATTTCCTCTTAGAACTCAATAG- $3^{\prime}$ ) and mus_macf1_1 reverse (5'-TGATCAAGACAACATCCTGTTTCTG-3'), mus_macf1_2 forward (5'-ACCAAGTACCTGTAGAAAAGCTTCA-3') and mus_macf1_2 reverse (5'-GCAGCTTGTTGGAGAGCTCCTC-3'), or mus_macf1_3 forward (5'-GGCAGAATTAAGCTGCCTCAAGG-3') and mus_macf1_3 reverse (5'-CAAGTTCAGTCCGGGTCATCGG-3'). Primers to amplify macfla cDNA (NCBI reference sequence XP_003200667.1) from zebrafish macular cDNA included zf_macfla_8 forward (5'-CCCAGTCTCATTCTGGACACTGT- $\left.{ }^{\prime}\right)$ and zf_macfla_8 reverse (5'-TTCCTCCAGCTTGTGTTGCCTCT- $3^{\prime}$ ). Primers to amplify macfl $1 b$ cDNA (NCBI reference sequence XM_690777.5) were Macf1b_F1 (5'-CGTTGCTGCGCAGGAGTTTCTCTG-3') and Macflb_R1 (5'-CTCAAAGCAGGACTCAAATCCAGTCGGTTC-3'). For negative controls, water was used in lieu of cDNA. PCR products were confirmed by DNA sequencing.

\section{$\leftarrow$}

(Figure legend continued.) C, RT-PCR demonstrates that Macf1 is expressed in mouse vestibular tissue using three primer pairs across the coding sequence of Macf1: RT plus (lanes 1, 3,5) and RT minus controls (lane 2, 4, 6). D-F, RNA in situ hybridization. Antisense probed 5-dpf zebrafish demonstrate that macfla is expressed in the otocyst (arrowhead, $\boldsymbol{D}$ ). Magnified images of otocysts reveal macfla expression in hair cells of the anterior macula (AM) and posterior crista $(P C)(\boldsymbol{E})$ but low background in control $(\boldsymbol{F})$. In the lateral line, macfla expression was below the level of confident detection by whole-mount in situ hybridization. G, The macf1a locus contains 97 exons (red bars), and the point of insertion (orange arrow) for the FlipTrap vector in the Gt(macf1a-citrine ${ }^{c t 68 a}$ strain is shown. TE, Transposable element; SA, splice acceptor; SD, splice donor. $\boldsymbol{H}$, The major functional domains of Acf7a and the position of Citrine. $\mathrm{CH}$, Calponin homology domain; GAR, GAS2-related domain. I-N, Images of Gt(macf1a-

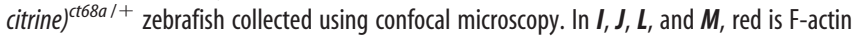
labeled with phalloidin. Hair cells expressing Acf7a-Citrine (yellow) have fusion protein encircling the $\mathrm{CP}$ and the fonticulus in an anterior lateral-line neuromast $(\boldsymbol{I}-\boldsymbol{K})$.J, Enlarged view of $\boldsymbol{I}$. $\boldsymbol{K}$, Localization of Acf7a-Citrine in four hair cells. $L$, A3D reconstruction of hair cells from a lateral crista demonstrates that Acf7a is concentrated toward the basal portions of the CPs. Hair bundles (arrows) and CPs (brackets) are marked in $\boldsymbol{L}$ and $\boldsymbol{M}$. In $\boldsymbol{M}$, the volume of red-yellow overlap from image $\boldsymbol{L}$ is pseudocolored in white. $\boldsymbol{N}$, Immunolabeled microtubules (red) of lateral crista hair cells demonstrate that Acf7a-Citrine is located toward the ends of the microtubules, proximal to the apical surfaces of the cells. Arrowheads indicate kinocilia. Each $c$, $f$, or $n$ denotes the position of a $(P$, fonticulus, or nucleus, respectively. Scale bars: $E, F, 5 \mu \mathrm{m} ; I-L, N, 2 \mu \mathrm{m}$.
Plasmid construction. A macfla cDNA segment was amplified by PCR using zebrafish macular cDNA. Primers used included zf_Macfla_Forward1 (5' ${ }^{\prime}$ TCACGTGTGATGGACTTCTTCAGA-3') and zf_Macfla_Reverse1 (5'-TCACTTCTTGCCTGCGGGTGTTT-3'). The product was subcloned into pCRII-TOPO (Invitrogen) to generate pCRII:ACF7a.

Zebrafish $\beta$-actin cDNA was amplified with primer pairs XmaI_beta_ actin (5'-CCCGGGATGGATGATGAAATTGCCGCACT-3') and PacI_ beta_actin ( $5^{\prime}$-TTAATTAAGAAGCATTTGCGGTGGACGATG-3'). The product was inserted into $\mathrm{pMT} / \mathrm{SV} / \mathrm{PV} 3 \mathrm{~b} / \mathrm{mCherry}$ (West and McDermott, 2011) to create $\mathrm{pMT} / \mathrm{SV} / \mathrm{PV} 3 \mathrm{~b} / \beta$-actin/mCherry.

RNA in situ hybridization. In situ hybridizations were performed (McDermott et al., 2007) using sense and antisense probes synthesized from pCRII:ACF7a.

Fish labeling and imaging. Gt(macf1a-citrine $)^{c t 68 a /+}$ zebrafish at $5 \mathrm{~d}$ post-fertilization (dpf) were immunolabeled with anti-acetylated tubulin (6-11B 1; Sigma) at a 1:1000 dilution and/or labeled with Alexa Fluor 546 phalloidin (Invitrogen) at a 1:50 dilution (Chou et al., 2011; West and McDermott, 2011). When appropriate, Alexa Fluor 405-labeled goat anti-mouse IgG (Invitrogen), at a 1:200 dilution, was used as secondary antibody. Images were acquired by confocal microscopy with a $40 \times$, $63 \times$, or $100 \times$ objective (Leica Confocal Software; Leica).

Zebrafish transgenesis. To generate transgenic zebrafish, plasmid pMT/ $\mathrm{SV} / \mathrm{PV} 3 \mathrm{~b} / \beta$-actin/mCherry at $100 \mathrm{ng} / \mu \mathrm{l}$ was coinjected with Tol2 RNA at $25 \mathrm{ng} / \mu \mathrm{l}$ (Balciunas et al., 2006) into embryos at the single-cell stage.

Labeling of mouse tissue. Maculae of mice, between 6 and 12 months of age, were labeled as described previously (Pataky et al., 2004), except fixed for $1 \mathrm{~h}$, permeabilized for $2 \mathrm{~h}$, and blocked for $2 \mathrm{~h}$. Sections of cochlea from P2 mice were cultured essentially as described previously (Russell and Richardson, 1987). Primary antibodies, purified ACF7 antiserum (Kodama et al., 2003) and mouse monoclonal antibody to acetylated $\alpha$-tubulin (T6793; Sigma), were used at a 1:100 dilution. Secondary antibodies, Alexa Fluor 488 goat anti-rabbit IgG and Alexa Flour 405 goat anti-mouse IgG (Invitrogen), were used at a 1:200 dilution. Alexa Fluor 568 phalloidin (Invitrogen) was used at a 1:50 dilution. Mounting medium (Vectashield; Vector Laboratories) was used when capturing images on a confocal microscope with a $40 \times$ or $100 \times$ oil-immersion objective. All labeling with ACF7 antibody was performed in parallel with secondary antibody alone, and no significant labeling was detected (data not shown).

$Z$-stacks of confocal sections, each $0.122 \mu \mathrm{m}$, through labeled tissue were converted into 3D models (Volocity; PerkinElmer). In Figure $2 H-L$, the green channel is amplified two times. In addition, the rendering software was used to identify regions of overlap between the signals. The non-overlapping signal was removed, and the overlapping signal was amplified (Fig. $2 G, 2.5 \times ; M, 8 \times$ ).

Electron microscopy and tomography. Anesthetized 5-dpf zebrafish were fixed in $0.1 \mathrm{~m}$ sodium cacodylate buffer, $\mathrm{pH} 7.2$, containing $2 \%$ paraformaldehyde and $0.1 \%$ glutaraldehyde and then processed by progressive lowering of temperature (Auer et al., 2008) before embedding in LR Gold (EMS). Tilt series were recorded at $\sim 1 \mu \mathrm{m}$ underfocus on a FEI Tecnai T12 using 120-nm-thick sections with a Gatan 1000 CCD set to collect at $2048 \times 2048$ pixels with $0.8 \mathrm{~nm} /$ pixel. $3 \mathrm{D}$ volumes were reconstructed and filtered with IMOD eTomo and Clip programs, respectively. Amira 5.3 software (VSG/FEI Visualization Sciences Group) was used for manual segmentation and surface rendering.

\section{Results}

Microtubule and actin crosslinking factor 1 genes are expressed in hair cells of fish, birds, and mammals

To identify candidate cytoskeletal proteins that may play key roles in establishing the unique morphology of the hair cell, we determined the hair-cell transcriptome by performing RNA-seq on manually isolated hair cells from the chicken. MACF1 mRNA was highly represented (Fig. 1A). In fact, when compared with other genes known to be specifically expressed in hair cells, such as protocadherin 15 (PCDH15) (Alagramam et al., 2001), MACF1 mRNA was more abundant. We calculated the reads per kilobase of transcript per million mapped reads (RPKM) for both 


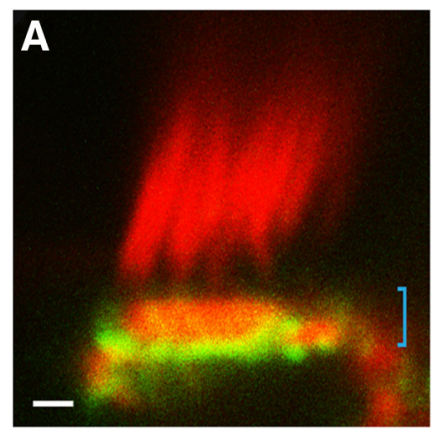

E
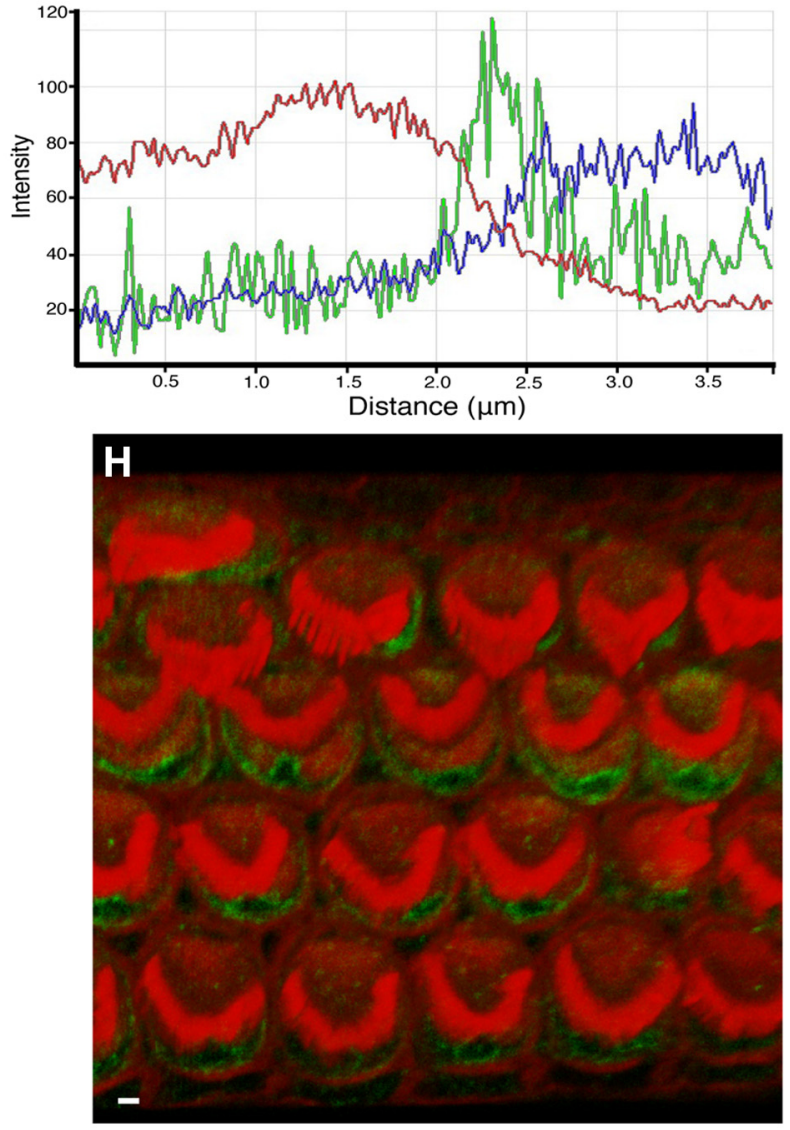

$\mathbf{J}$

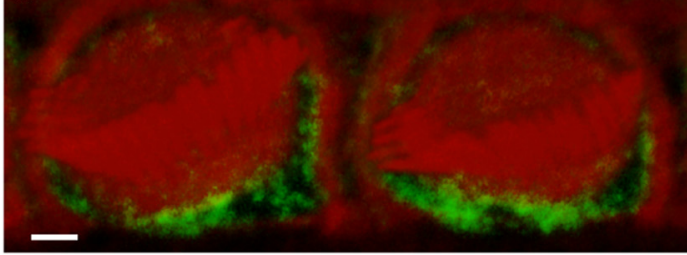

$\mathbf{L}$

L

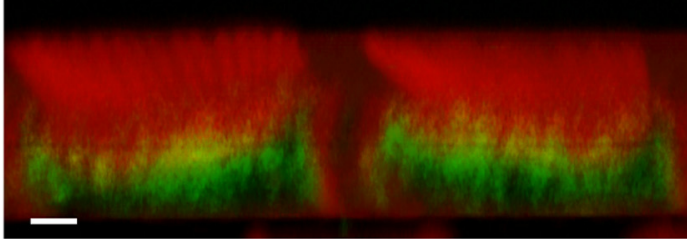

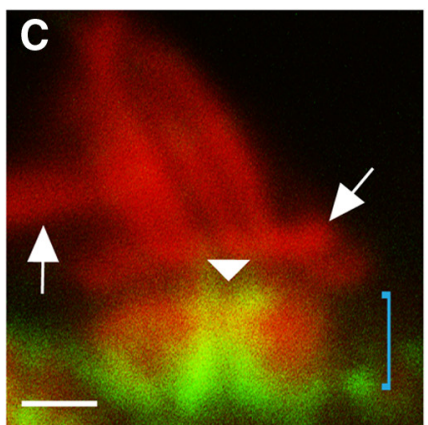
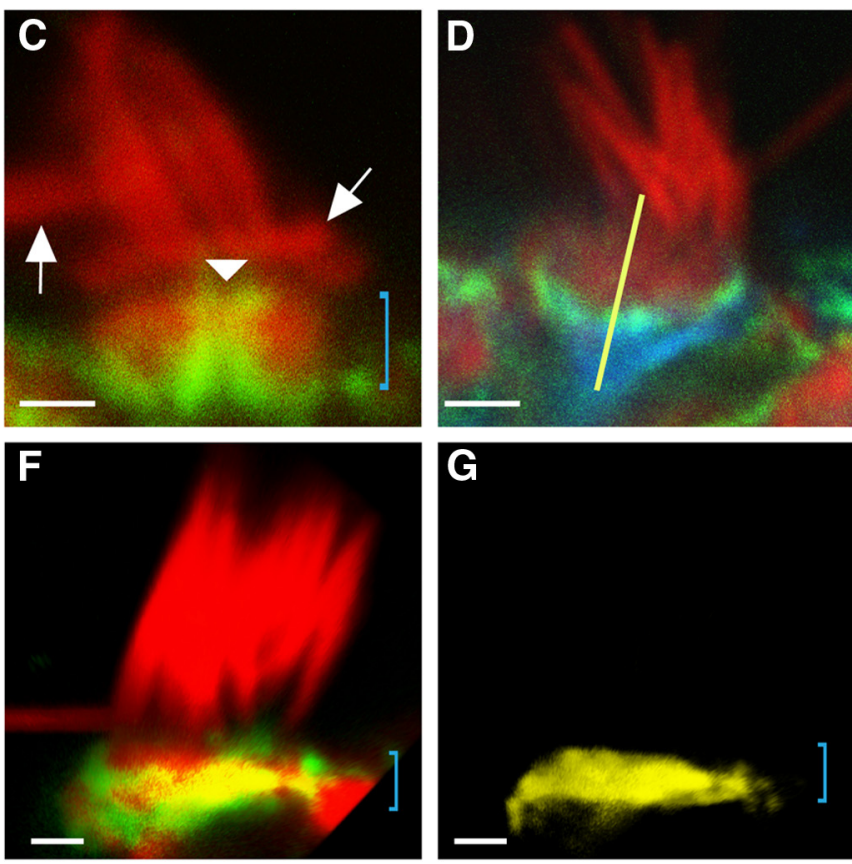

I
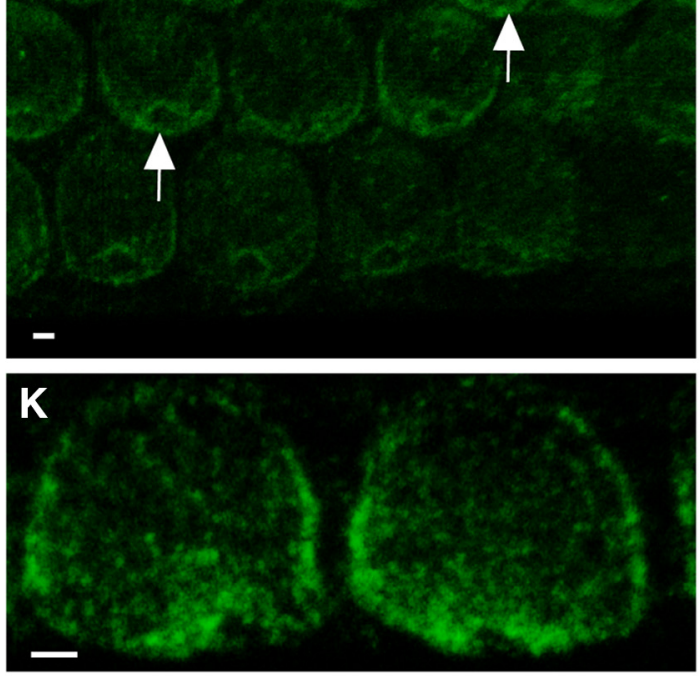

M

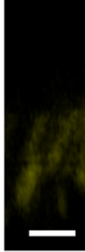

Figure 2. ACF7 distribution in mouse vestibular and cochlear hair cells. $A-D, F-M$, Images acquired using confocal microscopy. A-D, F, G, Adult mouse vestibular hair cells labeled with ACF7 antibody (green) and phalloidin (red).A, ACF7 is localized toward the base of the CP (bracket). B, ACF7 encircles the CP and forms a ring within the fonticulus. C, ACF7 coats the lumen of the fonticulus (arrowhead). Bracket denotes (P, above which are stereocilia with normal morphology and a few bent stereocilia (arrows). D, Cell colabeled with tubulin antibody (blue). $\boldsymbol{E}$, Fluorescence intensity profile from hair cell in $\boldsymbol{D}$, using the yellow-line region of interest (ROI), demonstrates that ACF7-associated signal (green) rests between phalloidin-associated (red) and tubulin-associated (blue) signals. Intensity scale is linear, but the units are arbitrary. $X$-axis represents the length of the ROl, from top $(0 \mu \mathrm{m})$ to bottom $(4 \mu \mathrm{m})$. $\boldsymbol{F}$, In $3 \mathrm{D}$ reconstruction, (Figure legend continues.) 

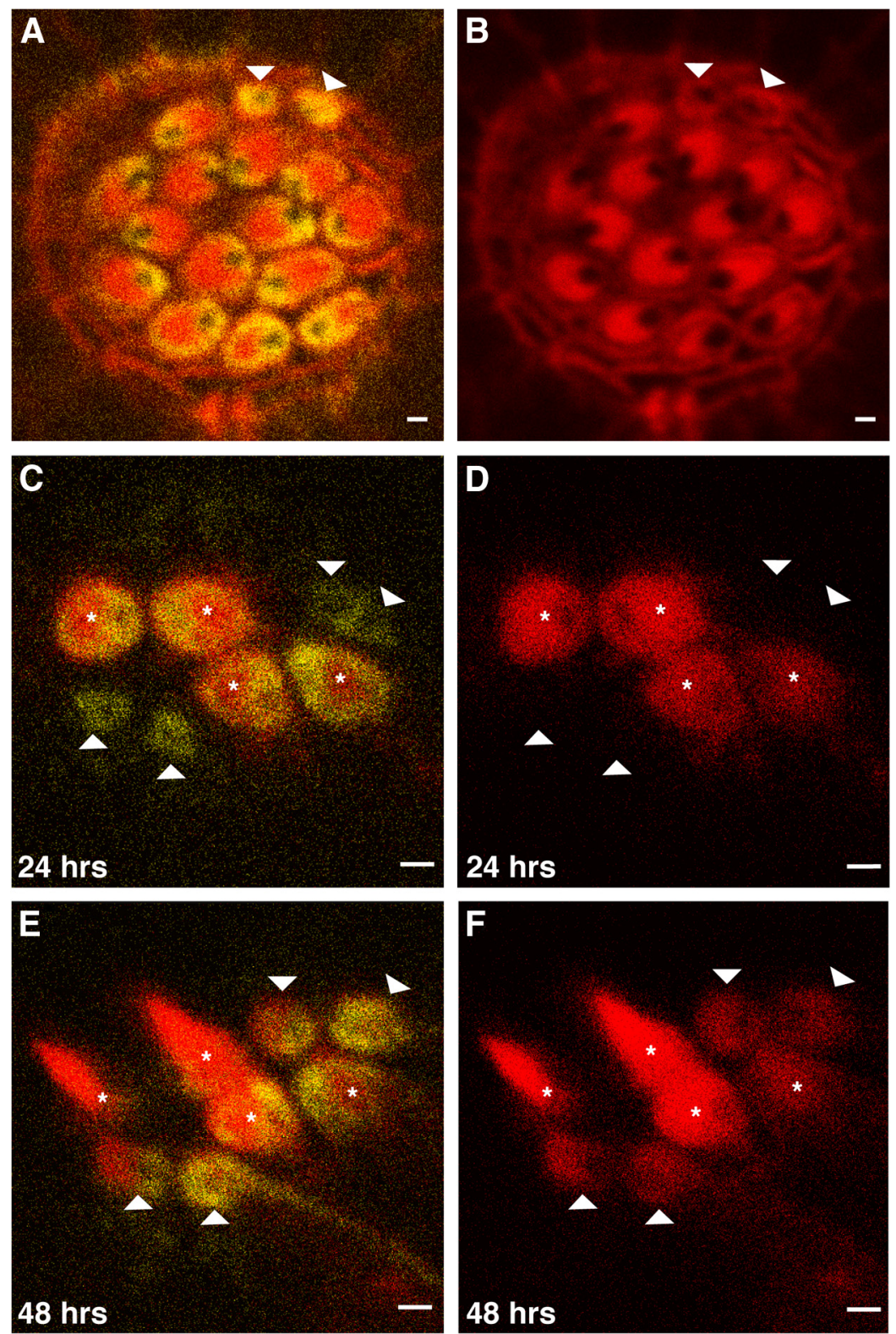

Figure 3. Apical localization of Acf7a precedes hair-bundle and CP formation. Micrographs collected using confocal microscopy. $A$, Hair cells of a posterior lateral-line neuromast, from a zebrafish at $5 \mathrm{dpf}$, expressing Acf7a-Citrine (yellow) are labeled with phalloidin (red). $\boldsymbol{B}$ displays only the red channel of $\boldsymbol{A}$. The CPs of the hair cells are oriented opposite to each other. Two cells ( $\boldsymbol{B}$, arrowheads) within the rosette lack significant actin labeling at their apical surfaces, indicating lack of hair bundles and (PS, but contain robust levels of Acf7a-Citrine (A). C, D, A live neuromast expressing Acf7a-Citrine and $\beta$-actin-mCherry $24 \mathrm{~h}$ after neomycin treatment. $\beta$-Actin-mCherry of the $C P$ and hair bundle is red, and Acf7a-Citrine is yellow. $\boldsymbol{D}$ displays red channel only. Four hair cells have regenerated and contain both Acf $\mathrm{a}$-Citrine and $\beta$-actin-mCherry at apical regions (asterisk); however, there are cells within the rosette that contain Acf7a-Citrine and no significant amounts of $\beta$-actin-mCherry (arrowheads). $\boldsymbol{E}_{\boldsymbol{F}} \boldsymbol{F}, \mathrm{At} 48 \mathrm{~h}$ after neomycin treatment, the cells that lacked apical localization of $\beta$-actin-mCherry developed CPs with PCP, confirming their status as hair cells (arrowheads). $\boldsymbol{F}$ displays red channel only. Scale bar, $1 \mu \mathrm{m}$.

(Figure legend continued.) yellow indicates volume of red and green overlap. Bracket indicates position of CP. G, The volume of overlap from $\boldsymbol{F}$ with non-overlapping signals removed. No significant differences in ACF7 localization were noted between type I and type II hair cells. $H-M, 3 D$ reconstructions of P2 mouse cochlear hair cells labeled with ACF7 antibody (green) and phalloidin (red). $\boldsymbol{H}, \boldsymbol{I}$, Images of a basal turn of a cochlea demonstrate that ACF7 forms a ring around each fonticulus and labeling is appreciable in and around each CP. Each arrow points out a typical fonticulus. Moreover, increased ACF7 levels are observed closer to the fonticulus. Labeling of cells in the bottom row is out of the plane of focus. $J-M$, Two inner hair cells. $L$, Image of two hair cells, from a lateral perspective, demonstrates that ACF7 resides basal to, and within, the CP. Yellow indicates overlapping signals. $\boldsymbol{M}$, Yellow signal from $L$ demonstrates that ACF7 overlaps with actin at the $C P$ and at a restricted region near the cortical surface of the hair cell (arrowhead). Scale bar, $1 \mu \mathrm{m}$.
MACF1- and PCDH15-associated reads. Single-end read counts yielded RPKM values of 6.02 and 2.09 for MACF1- and $P C D H 15$-associated signals, respectively. Next, we established that macfla and Macf1 mRNAs are expressed in maculae of genetically tractable vertebrates (zebrafish and mice, respectively) by RTPCR (Fig. 1B,C). By RNA in situ hybridization on zebrafish, it was demonstrated that all cristae and maculae express macf1a mRNA (Fig. 1D-F; data not shown). Finally, because zebrafish sometimes have two paralogous genes because of an antediluvian gene duplication event (Postlethwait et al., 2000), we searched the zebrafish genome and identified macf $1 b$ on chromosome 16 . We determined that this paralog is expressed in maculae of zebrafish by RT-PCR (Fig. 1B).

\section{Acf7a encircles the fonticulus and circumscribes, underlies, and is interwoven into the $\mathrm{CP}$ of zebrafish}

To determine the localization pattern of Acf7a in hair cells, we obtained a zebrafish strain that has the FlipTrap vector inserted in macfla on chromosome 19, allele Gt(macfla-citrine) ${ }^{c t 68 a}$ (Trinh le et al., 2011). Integration occurred in the intron between exon 57 and exon 58 (Fig. 1G), resulting in the expression of Acf7a fused to Citrine protein (Fig. $1 H$ ) driven by the endogenous macfla promoter. We fixed 5-dpf Gt(macfla-citrine $)^{\text {ct } 68 a /+} \mathrm{ze}$ brafish and labeled their F-actin with fluorophore-coupled phalloidin. Confocal micrographs demonstrated that Acf7a-Citrine circumscribes the $\mathrm{CP}$ of hair cells and forms a ring-like pattern around the fonticulus, which is known to contain the basal body (Fig. $1 I-K$ ). 3D reconstructions of images demonstrated that Acf7a-Citrine signal overlapped with actin-associated signal in the CP (Fig. 1 $L, M)$. Moreover, CPs were underlain by fusion protein (Fig. $1 L, M)$. Because ACF7 has been shown to bind microtubules, we determined the localization pattern of Acf7a-Citrine in relation to tubulin in hair cells. Immunolabeling of tubulin in Gt(macfla-citrine $)^{c t 68 a /+}$ zebrafish demonstrated that Acf7a-Citrine is apical to tubulin in hair-cell somata (Fig. 1N), excluding the area around the basal body that lacks Acf7a (Fig. 1J). These studies suggest that Acf7a is positioned to integrate these cytoskeletal networks, sandwiched between the $\mathrm{CP}$ actin, which is more apical, and somatic tubulin, which is more basal.

Localization of ACF7 in mammalian vestibular and auditory hair cells

Because the primary amino acid sequences of actin- and microtubule-binding domains of zebrafish Acf7a and mouse 
ACF7 are $>93 \%$ similar, we hypothesized that these proteins would localize similarly in these different species. Labeling using ACF7 antiserum and phalloidin revealed that, in mouse vestibular hair cells, ACF7 is adjacent to the basal face of the $\mathrm{CP}$ (Fig. 2A), is around the circumference of the $\mathrm{CP}$, and forms a ring around the fonticulus (Fig. 2B). Additionally, ACF7 coats the actin wall of the fonticulus (Fig. $2 C$ ). Fluorescence intensity profiles (Fig. $2 D, E$ ) revealed that ACF7 is couched between $\mathrm{CP}$ actin and immunolabeled microtubules. We also noticed faint but consistent labeling in the $\mathrm{CP}$ with antiACF7 antibody (Fig. 2A), the extent of which was revealed by $3 \mathrm{D}$ reconstructions (Fig. 2F, G).

To establish the localization pattern of ACF7 in auditory hair cells, organotypicexplant cultures from cochleae of $\mathrm{P} 2$ mice were labeled with ACF7 antiserum and phalloidin. 3D reconstructions show inner and outer hair cells each have a ring of ACF7 around their respective fonticulus, and the level of ACF7 labeling decreases with distance from each fonticulus (Fig. $2 H-K)$. ACF7 also extended below the $\mathrm{CP}$, with a modest amount intercalated into the $\mathrm{CP}$, and localized to a restricted region near the cortical surface toward the apex of the hair cell (Fig. $2 L, M$ ).

Acf7a is a hair bundle and CP antecedent We next endeavored to determine at what stage of hair-cell development Acf7a is positioned near the apical surface. Because the neuromast organ of zebrafish replaces hair cells throughout the life of the animal, we analyzed neuromasts of phalloidin-labeled Gt(macfla-citrine $)^{c t 68 a /+}$ 5-dpf embryos. Some cells contained apically localized Acf7a-Citrine but did not have CPs or hair bundles (Fig. $3 A, B$ ). Because these labeled cells were near hair cells within the rosette, we hypothesized that these cells were supporting cells, differentiating into hair cells, and Acf7a antecedes CP and hair-bundle formation.

To confirm that these cells are presumptive hair cells and not a supporting cell subpopulation that harbors Acf7a-Citrine, we generated a stable transgenic zebrafish strain that expresses mCherry fused to $\beta$-actin under the parvalbumin $3 b$ promoter (McDermott et al., 2010). This promoter is active in hair cells and supporting cells, which are transitioning to hair cells. This novel strain, $\operatorname{Tg}$ (pvalb3b: $\beta$-actin-mCherry), labels stereocilia and CPs of the lateral line with fusion protein, as confirmed by phalloidin labeling (data not shown), enabling observation of development in live animals.

For spatiotemporal analysis of hair-bundle formation relative to Acf7a localization, we performed a hair-cell regeneration assay of the CP by ACF7.
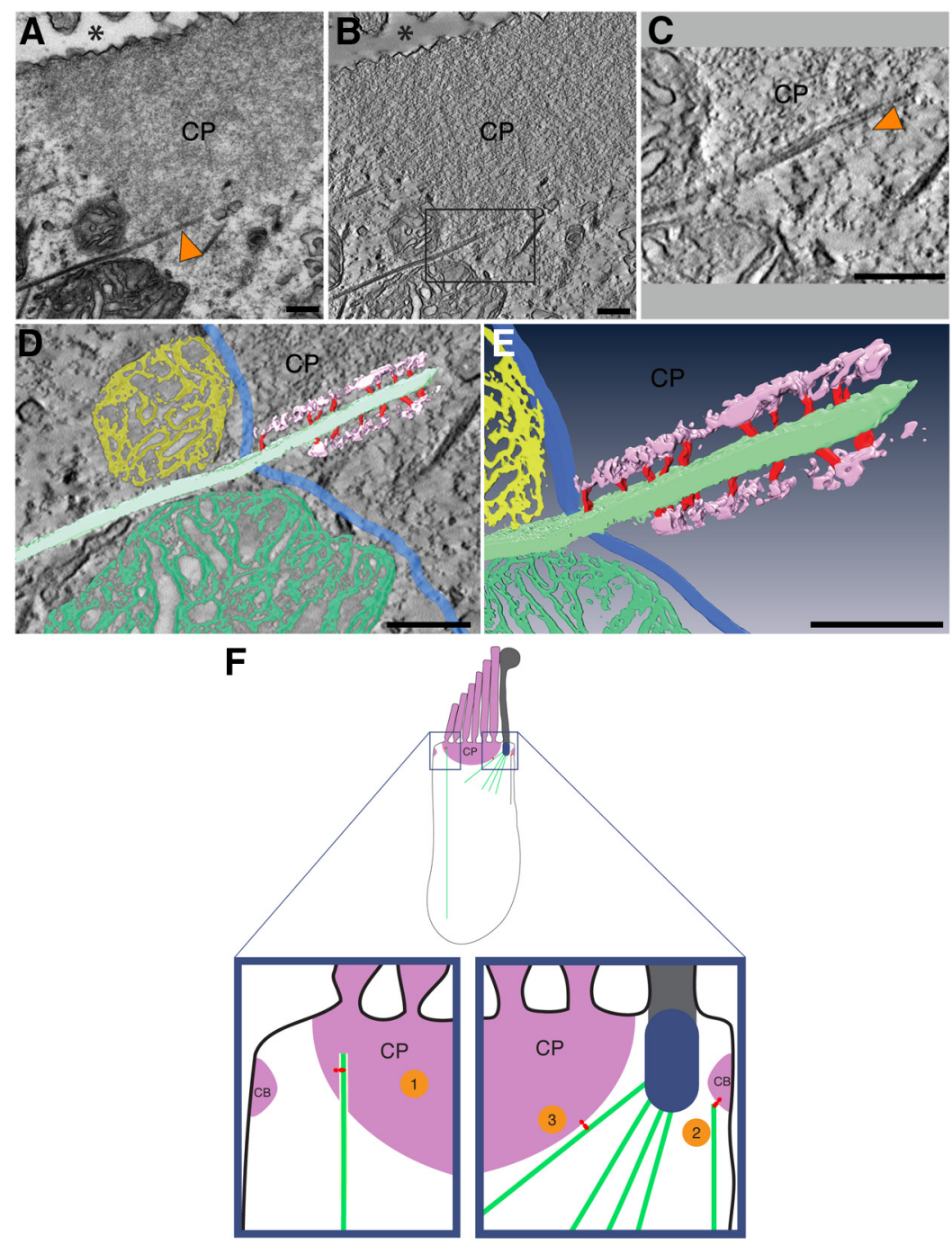

Figure 4. Microtubules inserted into the $(P$ are bridged to the actin meshwork via filamentous linkers. $A, 2 D$ transmission electron microscopy of an inner ear hair cell from a 6-dpf zebrafish reveals a microtubule (arrowhead) terminating within the CP. Asterisk shows position of the apical surface of the cell. $B, A \sim 1$-nm-thick slice through a tomographic 3D reconstruction corresponding to the $2 D$ projection view shown in $\boldsymbol{A}$. $\boldsymbol{C}$, An enlarged view of rectangle in $\boldsymbol{B}$. Arrowhead indicates a filamentous connection, which bridges the gap between the microtubule and actin. $\boldsymbol{D}, \boldsymbol{E}$, Manual segmentation and surface rendering of the densities of interest, with microtubule depicted in mint, actin meshwork in purple, and connecting filaments in red, revealing a $\sim 15$ - to 20-nm-wide gap between the microtubule and the actin meshwork, which is bridged by the filamentous connections. Boundary of the actin meshwork is indicated in blue. Mitochondria adjacent to the $C P$ are shown in yellow and green. $D$, Surface rendering of the segmented volume overlaid onto a central section through the 3D reconstruction. $\boldsymbol{E}$ contains only the segmented volume from $\boldsymbol{D}$. Scale bars, $200 \mathrm{~nm}$. $\boldsymbol{F}$, Models of ACF7 function in the mature hair cell. Top, Image of a hair cell. CP and circumferential band (CB) are represented as solid purple structures. Blue structure is the basal body, which resides in the fonticulus. ACF7 is shown in red. Model 1, ACF7 links a microtubule (mint), which penetrates into the CP, with the F-actin of the CP. Model 2, ACF7 links $\mathrm{F}$-actin of the circumferential band to a microtubule. Model 3, A microtubule that emanates from the basal body is joined to F-actin

(Murakami et al., 2003). We crossed Gt(macfla-citrine) $)^{\text {ct68a/+ }}$ and $\operatorname{Tg}$ (pvalb3b: $\beta$-actin-mCherry) to generate double transgenic offspring. At $7 \mathrm{dpf}$, neuromast hair cells were ablated with neomycin, and live imaging was performed $24 \mathrm{~h}$ after exposure. A few hair cells regenerated and matured to have $\beta$-actin-mCherrylabeled CPs with a ring of Acf7a-Citrine encircling each CP and each fonticulus (Fig. $3 C, D$ ). It was also noted that a few cells within the rosette had Acf7a-Citrine near their apical surfaces but with no observable $\beta$-actin-mCherry in the same regions (Fig. $3 C, D)$. After an additional $24 \mathrm{~h}$, i.e., $48 \mathrm{~h}$ after neomycin exposure, these cells matured into hair cells with identifiable hair bundles and CPs (Fig. 3E,F) and also retained Acf7a-Citrine 
localization. These findings combined demonstrate that, in addition to being maintained near the apical region in the mature hair cell, Acf7a precedes hair-bundle and CP formation during haircell development and regeneration.

\section{Electron tomography reveals filamentous linkers that couple microtubules to the actin meshwork of the $\mathrm{CP}$}

To determine whether there are linkers that join the $\mathrm{CP}$ actin to the microtubules near the apical surface of the hair cell, we used electron tomography. This technique has been successful at revealing the architecture of other regions of the hair cell (Lenzi et al., 1999; Auer et al., 2008; Fridberger et al., 2009; Vranceanu et al., 2012), and it may allow the identification of linkers. 2D transmission electron microscopy of sections of zebrafish inner ear hair cells confirms incidences of insertions of microtubules into the CP (Fig. $4 A$ ). In addition, the area surrounding the microtubule appeared slightly less electron dense than the surrounding CP (Fig. 4B). Using 3D tomographic reconstruction, we determined this gap to be $\sim 15-20 \mathrm{~nm}$ (Fig. $4 C$ ). A number of filamentous connections bridge this gap, providing links between the microtubule and the $\mathrm{CP}$ actin matrix (Fig. 4C). Manual segmentation focusing on the high-contrast densities allows the visual extraction of the features of interest, ultimately revealing the structure of the linkers (Fig. $4 D, E$ ). The depicted connections likely represent an underestimation because we did not include connections that appeared at lower contrast, possibly as a result of staining inhomogeneities. Filaments between the microtubule and the actin meshwork are elucidated, and they may correspond to Acf7a. These results provide well defined evidence of filamentous connections between microtubules and the actin meshwork of the CP.

\section{Discussion}

\section{ACF7 links CP actin to the microtubule network}

Ultrastructural studies have established that microtubules insert into the CP to link the plate to the rest of the soma of the hair cell (Jaeger et al., 1994). Our results reveal direct filamentous links between the microtubules and the CP (Fig. 4); however, the identities, properties, and regulation of such linker proteins have yet to be described. Here we demonstrate that, in hair cells, ACF7 is positioned between the $\mathrm{F}$-actin of the $\mathrm{CP}$ and the microtubules of the soma, a pattern conserved between fish and mammals. Because ACF7 harbors both actin- and microtubule-binding domains, this provides strong evidence to suggest that ACF7 serves as a major linchpin between microtubules and CP actin (Fig. $4 F$, model 1). It is also possible that ACF7 links microtubules to the circumferential band (Fig. 4F, model 2); this notion is supported by ACF7-actin colocalization near the apical portion of the lateral membrane in mouse hair cells (Fig. $2 L, M$ ). Given the robust labeling of ACF7 around the fonticulus, it is possible that the microtubules emanating from the basal body (Slepecky et al., 1981) are tethered to the CP by ACF7 (Fig. 4F, model 3). In addition, ACF7 may help absorb and distribute the forces experienced during mechanotransduction through numerous spectrin repeats, which are thought to endow ACF7 with elasticity (Suozzi et al., 2012).

\section{The significance of ACF7 in hair-cell polarity}

Our findings demonstrate that Acf7a precedes the hair bundle and CP at the apical surface of the presumptive hair cell. ACF7 has been shown to bind and guide the growing end of microtubules in endodermal cells (Kodama et al., 2003), and, in epidermal cells, ACF7 promotes the coordinated tracking of mi- crotubules along actin fibers that converge at integrin-mediated focal adhesions, the polarized front of a wound edge (Wu et al., 2011). Microtubules and associated proteins are able to specify and maintain the site of cortical actin polarity in various cell types by delivering positional information to the cortex and inducing local actin polymerization. Therefore, ACF7 may play a role in targeting microtubules to the cell cortex to participate in hair-cell apical-basal polarity and may persist to assist in organizing the actin of the CP.

In the organ of Corti and the vestibular epithelia, hair cells display planar cell polarity (PCP). Wnt signaling has been implicated in the development of the inner ear (Montcouquiol et al., 2006; Sienknecht and Fekete, 2009) and in the establishment of PCP of outer hair cells (Dabdoub et al., 2003). Interestingly, the microtubule binding capacity of ACF7 is controlled through phosphorylation by glycogen synthase kinase $3 \beta$, whose activity is inhibited by Wnt signaling (Wu et al., 2011). The distribution of ACF7 around the CP is asymmetrical (Fig. $2 \mathrm{H}-\mathrm{K}$ ), reminiscent of other proteins known to be involved in PCP (Wang et al., 2005). Therefore, the spatial and temporal regulation of ACF7 by Wnt signaling may be involved in establishing PCP in hair cells. Finally, we noted that ACF7 localized to a restricted region near the cortical surface toward the apex of the hair cell (Fig. $2 L, M$ ). This junctional region of the hair cell is part of the reticular lamina, which contains both tight junctions and adherence junctions. The reticular lamina serves two major functions in the ear. It separates compartments of the cochlea that have significantly different ionic contents, the endolymph and perilymph (Gulley and Reese, 1976). In addition, the reticular lamina is capable of enduring the mechanical stress exacted by sound-evoked stimulation (Fridberger et al., 2002). Therefore, ACF7 may play a role in formation or maintenance of this vital structure.

\section{References}

Alagramam KN, Murcia CL, Kwon HY, Pawlowski KS, Wright CG, Woychik RP (2001) The mouse Ames waltzer hearing-loss mutant is caused by mutation of Pcdh15, a novel protocadherin gene. Nat Genet 27:99-102. CrossRef Medline

Auer M, Koster AJ, Ziese U, Bajaj C, Volkmann N, Wang da N, Hudspeth AJ (2008) Three-dimensional architecture of hair-bundle linkages revealed by electron-microscopic tomography. J Assoc Res Otolaryngol 9:215224. CrossRef Medline

Balciunas D, Wangensteen KJ, Wilber A, Bell J, Geurts A, Sivasubbu S, Wang X, Hackett PB, Largaespada DA, McIvor RS, Ekker SC (2006) Harnessing a high cargo-capacity transposon for genetic applications in vertebrates. PLoS Genet 2:e169. CrossRef Medline

Chacon-Heszele MF, Ren D, Reynolds AB, Chi F, Chen P (2012) Regulation of cochlear convergent extension by the vertebrate planar cell polarity pathway is dependent on p120-catenin. Development 139:968-978. CrossRef Medline

Chen HJ, Lin CM, Lin CS, Perez-Olle R, Leung CL, Liem RK (2006) The role of microtubule actin cross-linking factor 1 (MACF1) in the Wnt signaling pathway. Genes Dev 20:1933-1945. CrossRef Medline

Chou SW, Hwang P, Gomez G, Fernando CA, West MC, Pollock LM, LinJones J, Burnside B, McDermott BM Jr (2011) Fascin 2b is a component of stereocilia that lengthens actin-based protrusions. PLoS One 6:e14807. CrossRef Medline

Dabdoub A, Donohue MJ, Brennan A, Wolf V, Montcouquiol M, Sassoon DA, Hseih JC, Rubin JS, Salinas PC, Kelley MW (2003) Wnt signaling mediates reorientation of outer hair cell stereociliary bundles in the mammalian cochlea. Development 130:2375-2384. CrossRef Medline

DeRosier DJ, Tilney LG (1989) The structure of the cuticular plate, an in vivo actin gel. J Cell Biol 109:2853-2867. CrossRef Medline

Dosch R, Wagner DS, Mintzer KA, Runke G, Wiemelt AP, Mullins MC (2004) Maternal control of vertebrate development before the midblastula transition: mutants from the zebrafish I. Dev Cell 6:771-780. CrossRef Medline 
Fridberger A, Boutet de Monvel J, Ulfendahl M (2002) Internal shearing within the hearing organ evoked by basilar membrane motion. J Neurosci 22:9850-9857. Medline

Fridberger A, Von Tiedemann M, Flock A, Flock B, Ofverstedt LG, Skoglund U (2009) Three-dimensional structure of outer hair cell pillars. Acta Otolaryngol 129:940-945. CrossRef Medline

Gulley RL, Reese TS (1976) Intercellular junctions in the reticular lamina of the organ of Corti. J Neurocytol 5:479-507. CrossRef Medline

Gupta T, Marlow FL, Ferriola D, Mackiewicz K, Dapprich J, Monos D, Mullins MC (2010) Microtubule actin crosslinking factor 1 regulates the Balbiani body and animal-vegetal polarity of the zebrafish oocyte. PLoS Genet 6:e1001073. CrossRef Medline

Hawkins JE Jr (1976) Drug ototoxicity. In: Handbook of sensory physiology, Vol V/3, Auditory system: clinical and special topics, Chap 16 (Keidel WD, Neff WD, eds), pp 707-748. Berlin: Springer.

Hudspeth AJ (1982) Extracellular current flow and the site of transduction by vertebrate hair cells. J Neurosci 2:1-10. Medline

Jaeger RG, Fex J, Kachar B (1994) Structural basis for mechanical transduction in the frog vestibular sensory apparatus: II. The role of microtubules in the organization of the cuticular plate. Hear Res 77:207-215. CrossRef Medline

Kodama A, Karakesisoglou I, Wong E, Vaezi A, Fuchs E (2003) ACF7: an essential integrator of microtubule dynamics. Cell 115:343-354. CrossRef Medline

Lenzi D, Runyeon JW, Crum J, Ellisman MH, Roberts WM (1999) Synaptic vesicle populations in saccular hair cells reconstructed by electron tomography. J Neurosci 19:119-132. Medline

Leung CL, Sun D, Zheng M, Knowles DR, Liem RK (1999) Microtubule actin cross-linking factor (MACF): a hybrid of dystonin and dystrophin that can interact with the actin and microtubule cytoskeletons. J Cell Biol 147:1275-1286. CrossRef Medline

McDermott BM Jr, Baucom JM, Hudspeth AJ (2007) Analysis and functional evaluation of the haircell transcriptome. Proc Natl Acad Sci U S A 104:11820-11825. CrossRef Medline

McDermott BM Jr, Asai Y, Baucom JM, Jani SD, Castellanos Y, Gomez G, McClintock JM, Starr CJ, Hudspeth AJ (2010) Transgenic labeling of hair cells in the zebrafish acousticolateralis system. Gene Expr Patterns 10:113-118. CrossRef Medline

Montcouquiol M, Crenshaw EB 3rd, Kelley MW (2006) Noncanonical Wnt signaling and neural polarity. Annu Rev Neurosci 29:363-386. CrossRef Medline

Murakami SL, Cunningham LL, Werner LA, Bauer E, Pujol R, Raible DW, Rubel EW (2003) Developmental differences in susceptibility to neomycin-induced hair cell death in the lateral line neuromasts of zebrafish (Danio rerio). Hear Res 186:47-56. CrossRef Medline

Nicol JW, Helt GA, Blanchard SG Jr, Raja A, Loraine AE (2009) The Integrated Genome Browser: free software for distribution and exploration of genome-scale datasets. Bioinformatics 25:2730-2731. CrossRef Medline

Pataky F, Pironkova R, Hudspeth AJ (2004) Radixin is a constituent of stereocilia in hair cells. Proc Natl Acad Sci U S A 101:2601-2606. CrossRef Medline
Postlethwait JH, Woods IG, Ngo-Hazelett P, Yan YL, Kelly PD, Chu F, Huang H, Hill-Force A, Talbot WS (2000) Zebrafish comparative genomics and the origins of vertebrate chromosomes. Genome Res 10:1890-1902. CrossRef Medline

Raphael Y, Altschuler RA (2003) Structure and innervation of the cochlea. Brain Res Bull 60:397-422. CrossRef Medline

Raphael Y, Athey BD, Wang Y, Lee MK, Altschuler RA (1994) F-actin, tubulin and spectrin in the organ of Corti: comparative distribution in different cell types and mammalian species. Hear Res 76:173-187. CrossRef Medline

Russell IJ, Richardson GP (1987) The morphology and physiology of hair cells in organotypic cultures of the mouse cochlea. Hear Res 31:9-24. CrossRef Medline

Sienknecht UJ, Fekete DM (2009) Mapping of Wnt, frizzled, and Wnt inhibitor gene expression domains in the avian otic primordium. J Comp Neurol 517:751-764. CrossRef Medline

Slepecky N, Hamernik R, Henderson D (1981) The consistent occurrence of a striated organelle (Friedmann body) in the inner hair cells of the normal chinchilla. Acta Otolaryngol 91:189-198. CrossRef Medline

Suozzi KC, Wu X, Fuchs E (2012) Spectraplakins: master orchestrators of cytoskeletal dynamics. J Cell Biol 197:465-475. CrossRef Medline

Tilney LG, Tilney MS, DeRosier DJ (1992) Actin filaments, stereocilia, and hair cells: how cells count and measure. Annu Rev Cell Biol 8:257-274. CrossRef Medline

Trapnell C, Williams BA, Pertea G, Mortazavi A, Kwan G, van Baren MJ, Salzberg SL, Wold BJ, Pachter L (2010) Transcript assembly and quantification by RNA-Seq reveals unannotated transcripts and isoform switching during cell differentiation. Nat Biotechnol 28:511-515. CrossRef Medline

Trinh le A, Hochgreb T, Graham M, Wu D, Ruf-Zamojski F, Jayasena CS, Saxena A, Hawk R, Gonzalez-Serricchio A, Dixson A, Chow E, Gonzales C, Leung HY, Solomon I, Bronner-Fraser M, Megason SG, Fraser SE (2011) A versatile gene trap to visualize and interrogate the function of the vertebrate proteome. Genes Dev 25:2306-2320. CrossRef Medline

Vranceanu F, Perkins GA, Terada M, Chidavaenzi RL, Ellisman MH, Lysakowski A (2012) Striated organelle, a cytoskeletal structure positioned to modulate hair-cell transduction. Proc Natl Acad Sci U S A 109:44734478. CrossRef Medline

Wang J, Mark S, Zhang X, Qian D, Yoo SJ, Radde-Gallwitz K, Zhang Y, Lin X, Collazo A, Wynshaw-Boris A, Chen P (2005) Regulation of polarized extension and planar cell polarity in the cochlea by the vertebrate PCP pathway. Nat Genet 37:980-985. CrossRef Medline

West MC, McDermott BM Jr (2011) Ribeye a-mCherry fusion protein: a novel tool for labeling synaptic ribbons of the hair cell. J Neurosci Methods 197:274-278. CrossRef Medline

Wu X, Shen QT, Oristian DS, Lu CP, Zheng Q, Wang HW, Fuchs E (2011) Skin stem cells orchestrate directional migration by regulating microtubule-ACF7 connections through GSK3 $\beta$. Cell 144:341-352. CrossRef Medline 